\title{
Solar energetic particle access to distant longitudes through turbulent field-line meandering
}

\author{
T. Laitinen ${ }^{1}$, A. Kopp ${ }^{2, \star}$, F. Effenberger ${ }^{3,4}$, S. Dalla ${ }^{1}$, and M. S. Marsh ${ }^{1, \star \star}$ \\ 1 Jeremiah Horrocks Institute, University of Central Lancashire, Preston, UK \\ e-mail: tlmlaitinen@uclan.ac.uk \\ 2 Université Libre de Bruxelles, Service de Physique Statistique et des Plasmas, CP 231, 1050 Brussels, Belgium \\ 3 Department of Mathematics, University of Waikato, Hamilton, New Zealand \\ ${ }^{4}$ Department of Physics and KIPAC, Stanford University, Stanford, CA 94305, USA
}

Received 21 November 2015 / Accepted 23 March 2016

\begin{abstract}
Context. Current solar energetic particle (SEP) propagation models describe the effects of interplanetary plasma turbulence on SEPs as diffusion, using a Fokker-Planck (FP) equation. However, FP models cannot explain the observed fast access of SEPs across the average magnetic field to regions that are widely separated in longitude within the heliosphere without using unrealistically strong cross-field diffusion.

Aims. We study whether the recently suggested early non-diffusive phase of SEP propagation can explain the wide SEP events with realistic particle transport parameters.

Methods. We used a novel model that accounts for the SEP propagation along field lines that meander as a result of plasma turbulence. Such a non-diffusive propagation mode has been shown to dominate the SEP cross-field propagation early in the SEP event history. We compare the new model to the traditional approach, and to SEP observations.

Results. Using the new model, we reproduce the observed longitudinal extent of SEP peak fluxes that are characterised by a Gaussian profile with $\sigma=30-50^{\circ}$, while current diffusion theory can only explain extents of $11^{\circ}$ with realistic diffusion coefficients. Our model also reproduces the timing of SEP arrival at distant longitudes, which cannot be explained using the diffusion model.

Conclusions. The early onset of SEPs over a wide range of longitudes can be understood as a result of the effects of magnetic fieldline random walk in the interplanetary medium and requires an SEP transport model that properly describes the non-diffusive early phase of SEP cross-field propagation.
\end{abstract}

Key words. Sun: particle emission - turbulence - Sun: heliosphere - magnetic fields - diffusion

\section{Introduction}

Solar energetic particles (SEPs) are accelerated up to relativistic energies during solar eruptions. Their propagation in interplanetary space is controlled by the large-scale Parker spiral magnetic field. SEPs can also propagate across the mean field as a result of large-scale drifts (Marsh et al. 2013) and the turbulent magnetic field fluctuations superposed on the mean field.

SEP propagation in interplanetary space is typically modelled by solving a Fokker-Planck equation that describes the effect of the plasma turbulence on the particles as diffusion (Parker 1965; Jokipii 1966). The field-aligned propagation is affected by small-scale inhomogeneities (Jokipii 1966), whereas the cross-field propagation is described as diffusion caused by random-walking field lines (Jokipii 1966; Matthaeus et al. 2003; Shalchi 2010). These theories give the ratio of the cross-field and field-aligned diffusion coefficients $\kappa_{\perp} / \kappa_{\|} \sim 0.01$ near Earth, and these values are supported by cosmic ray observations (Burger et al. 2000; Potgieter et al. 2014) and particle simulations (Giacalone \& Jokipii 1999). Recently, several works have studied the effect of cross-field diffusion on

\footnotetext{
^ Previously at the Institut für Experimentelle und Angewandte Physik, Christian-Albrecht-Universität zu Kiel, Germany.

$\star \star$ Now at Met Office, Exeter, UK.
}

SEP evolution (Zhang et al. 2009; Dröge et al. 2010; He et al. 2011; Giacalone \& Jokipii 2012; Qin et al. 2013) and showed up to $360^{\circ}$ heliolongitudinal extents for wide source regions with onsets within days of the SEP injection.

Recent multi-spacecraft SEP observations, made with the near-Earth spacecraft SOHO and ACE and the two STEREO spacecraft, show that SEPs from a single solar eruption have fast access to a very wide range of longitudes, even $180^{\circ}$ from the eruption location (e.g. Dresing et al. 2012; Richardson et al. 2014). Similar event extents as measured by longitudinal peak intensity distribution width have been observed both in gradual and impulsive events (Wiedenbeck et al. 2013; Cohen et al. 2014; Richardson et al. 2014), which in traditional view have very different acceleration origins (e.g. Reames 1999). Detailed analysis of two SEP multi-spacecraft-observed events, with SEP transport fitted with a 3D FP transport model, favour a small source region of about $20^{\circ}$ for the SEPs and a high diffusion coefficient ratio $\kappa_{\perp} / \kappa_{\|} \sim 0.1$ (Dresing et al. 2012; Dröge et al. 2014). High ratios in the range of $0.1-1$ have also been inferred in other studies of heliospheric particles (e.g. Zhang et al. 2003; Dwyer et al. 1997).

High values of $\kappa_{\perp} / \kappa_{\|}$are not supported by our current theoretical understanding of SEP transport in plasma turbulence, however. At $1 \mathrm{AU}$, the amplitude of transverse magnetic 
fluctuations, normalised with the background magnetic field, $\delta B_{\perp} / B$, is observed to be between 0.1 and 0.5 (e.g. Burlaga \& Turner 1976), for which full-orbit particle simulations suggest $\kappa_{\perp} / \kappa_{\|}<0.01$ Giacalone \& Jokipii (1999). Current theoretical understanding also supports much lower values for the ratio (e.g. Pei et al. 2010).

In this paper, we introduce a new model for heliospheric SEP propagation that is capable of reproducing the fast transport in longitude that is observed at the beginning of SEP events. Our work is based on the notion that SEPs spread initially much faster than allowed by a diffusion description, and that this is a result of field-line meandering (Laitinen et al. 2013). The crossfield propagation of SEPs along meandering fields has been analysed previously to study SEP intensity dropouts (Giacalone et al. 2000) and to determine diffusion coefficients (Kelly et al. 2012). Laitinen et al. (2013) showed for a uniform magnetic field that particle propagation across the mean field is initially a fast nondiffusive process along the meandering field-lines that only later relaxes to diffusion. Our new approach combines field-line meandering and time-asymptotic diffusion in a new description of early-time SEP propagation in the Parker field, the FP+FLRW model. We compare the new model to the traditional diffusion approach (the FP model).

\section{Models}

\subsection{FP model}

In the traditional approach, SEP propagation is solved using the Fokker-Planck equation for the distribution function $f$ (Roelof 1969; Skilling 1971; Isenberg 1997; Zhang et al. 2009)

$$
\begin{aligned}
& \frac{\partial f}{\partial t}+\left(\mu v \boldsymbol{b}+\boldsymbol{V}_{\mathrm{sw}}\right) \cdot \nabla f+\frac{v}{2 L}\left(1-\mu^{2}\right) \frac{\partial f}{\partial \mu} \\
& +\left[\frac{\mu\left(1-\mu^{2}\right)}{2}\left(\nabla \cdot \boldsymbol{V}_{\mathrm{sw}}-3 \boldsymbol{b} \boldsymbol{b}: \nabla \boldsymbol{V}_{\mathrm{sw}}\right)\right] \frac{\partial f}{\partial \mu} \\
& +\left[\frac{1-3 \mu^{2}}{2} \boldsymbol{b} \boldsymbol{b}: \nabla \boldsymbol{V}_{\mathrm{sw}}-\frac{1-\mu^{2}}{2} \nabla \cdot \boldsymbol{V}_{\mathrm{sw}}\right] p \frac{\partial f}{\partial p} \\
& =\frac{\partial}{\partial \mu}\left(D_{\mu \mu} \frac{\partial f}{\partial \mu}\right)+\nabla \cdot \hat{\kappa} \nabla f+Q(\boldsymbol{r}, \boldsymbol{v}, t)
\end{aligned}
$$

where $v, p$ and $\mu$ are the particle speed, momentum, and pitchangle cosine, $\boldsymbol{V}_{\mathrm{sw}}$ and $\boldsymbol{b}$ the solar wind velocity and a unit vector along the local mean magnetic field, respectively, and $L=-B /(\partial B / \partial s)$ the focusing length of the particles, with $s$ the arc length along the field line, and $Q$ is the injection function. The terms of the order $\left(\mathrm{d} V_{\mathrm{sw}} / \mathrm{d} t\right) / v$ (Isenberg 1997) are typically neglected in SEP transport studies as small compared to the other terms (e.g. Zhang et al. 2009; Dröge et al. 2010), and they vanish for the constant radial solar wind considered in our study (see Sect. 2.3).

The pitch-angle diffusion coefficient $D_{\mu \mu}$ and the crossfield diffusion coefficient $\kappa_{\perp}$ are given as (Jokipii 1966; Matthaeus et al. 2003)

$$
\begin{aligned}
& D_{\mu \mu}=\frac{\pi \Omega^{2}}{v|\mu| B^{2}}\left(1-\mu^{2}\right) S_{\|}\left(-\left(r_{\mathrm{L}} \mu\right)^{-1}\right) \\
& \kappa_{\perp}=\frac{a_{\mathrm{NLGC}}^{2} v^{2}}{3 B^{2}} \int \mathrm{d}^{3} k \frac{S(\boldsymbol{k})}{v / \lambda_{\|}+k_{\perp}^{2} \kappa_{\perp}+k_{\|}^{2} \kappa_{\|}},
\end{aligned}
$$

where $S(\boldsymbol{k})$ is the turbulence power spectrum, $S_{\|}$the spectrum of slab turbulence (see Sect. 2.3), $\Omega$ and $r_{\mathrm{L}}$ are the particle Larmor frequency and radius, respectively, the free parameter $a_{\mathrm{NLGC}}=$ $1 / \sqrt{3}$ (Matthaeus et al. 2003), and the parallel mean free path

$\lambda_{\|}=\frac{3 v}{4} \int_{0}^{1} \mathrm{~d} \mu \frac{\left(1-\mu^{2}\right)^{2}}{D_{\mu \mu}}$.

The field-aligned spatial diffusion tensor is given as

$\hat{\kappa}=\left(\begin{array}{ccc}\kappa_{\perp} & 0 & 0 \\ 0 & \kappa_{\perp} & 0 \\ 0 & 0 & 0\end{array}\right)$,

that is, without parallel diffusion, because this is accounted for with $D_{\mu \mu}$.

The changes in SEP energy during propagation in the interplanetary space are given by the terms in the third line of Eq. (1). They include momentum changes that are due to the diverging solar wind, and in an approximate way, the effect of drifts on the particle momentum (Dalla et al. 2015). However, the equation does not contain the drifts in its convective term (second term in the first line), which means that the transport picture presented by Eq. (1) is incomplete. The effect of latitudinal drifts on the particle energy was recently discussed by Dalla et al. (2015), who found that in 100 hours a $10 \mathrm{MeV}$ proton can lose almost half of its energy through drifting along the $\boldsymbol{V}_{\mathrm{sw}} \times \boldsymbol{B}$ electric field and adiabatic deceleration. An improved approach, the drift kinetic theory, introduces drifts within the convective term (e.g. le Roux \& Webb 2009; Webb et al. 2009).

The energy changes that are due to drifts and the diverging solar wind may be significant when performing a detailed fitting of the SEP event onset and the decay phase (e.g. Ruffolo 1995; Dalla et al. 2015). However, we here consider neither, but only the first ten hours of the event. For simplicity, the energy change term is therefore neglected in this study. Because the spatial transport associated with drift is small for a $10 \mathrm{MeV}$ proton within the early phase of an SEP event (Dalla et al. 2015), drifts are neglected within the convective term.

Particle diffusion parallel and across the mean field direction, as understood by current theories, are not independent of each other. The cross-field diffusion coefficient in Eq. (3) depends on the parallel diffusion coefficient, resulting in compound diffusion (e.g. Kóta \& Jokipii 2000; Qin et al. 2002; Matthaeus et al. 2003). The pitch angle dependence of the cross-field diffusion coefficient has also been discussed recently, with suggestions for using $\kappa_{\perp} \propto|\mu|$ (e.g. Qin \& Shalchi 2014) or $\kappa_{\perp} \propto r_{\mathrm{L}}$, the particle Larmor radius (Dröge et al. 2010). While the suggested different forms of pitch angle dependence of $\kappa_{\perp}$ influence particle cross-field propagation (e.g. Strauss \& Fichtner 2015), it has not been clearly established which type of dependence best represents particle behaviour in turbulent fields. We therefore use the conventional pitch-angle independent form of $\kappa_{\perp}$ here.

We solved Eq. (1) using stochastic differential equations (SDEs, e.g. Gardiner 2009), which solve the equations using pseudo-particles. The method is briefly described in Appendix A, further details are given in Kopp et al. (2012). We used enhanced pitch angle scattering across $\mu=0$ by using a pitch angle diffusion coefficient of the form

$D_{\mu \mu}=\kappa_{0}\left(|\mu|^{q-1}+H\right)\left(1-\mu^{2}\right)$,

suggested by Beeck \& Wibberenz (1986), where $\kappa_{0}$ contains the $\mu$-independent terms in Eq. (2), $q$ is the spectral index of the slab turbulence, and $H=0.1$ is a parameter that enhances scattering 
between pitch angle hemispheres. The FP equation is solved in a Parker spiral magnetic field with the magnitude $B$ given as

$B(r)=B_{0}\left(\frac{r_{0}}{r}\right)^{2} \sqrt{\frac{r^{2}+a^{2}}{r_{0}^{2}+a^{2}}}$,

where $B_{0}=5 \mathrm{nT}$ is the magnetic field at heliocentric distance $r_{0}=1 \mathrm{AU}$, and $a=V_{\mathrm{sw}} /\left(\Omega_{\odot} \sin \theta\right)$, where $V_{\mathrm{sw}}=400 \mathrm{~km} \mathrm{~s}^{-1}$, $\Omega_{\odot}=2.8631 \times 10^{-6} \mathrm{rad} / \mathrm{s}$ is the solar rotation rate and $\theta$ the colatitude.

\subsection{FP+FLRW model}

Laitinen et al. (2013) demonstrated that the early-time particle transport across the mean magnetic field is dominated by the particles propagating along meandering field lines. With respect to the mean magnetic field direction, the early-time cross-field propagation of the particles is therefore only weakly stochastic. At later times, Laitinen et al. (2013) showed that the cross-field extent of the particle population begins to widen, resulting in an asymptotic diffusive behaviour.

In this study, we used an FP+FLRW model that explicitly introduces the random walk of field lines across the Parker spiral magnetic field and particle propagation along these randomwalking field lines. A similar model was successfully used by Laitinen et al. (2013) for Cartesian geometry. In addition to propagating particles along meandering field lines, the particles are diffused across them with the time-asymptotic cross-field particle diffusion coefficient $\kappa_{\perp}$ to facilitate the late-time widening of the particle population. Laitinen et al. (2013) showed that such a model reproduces both the initial wide extent of the SEP event (where the FLRW dominates) and the asymptotic diffusive behaviour at later times (where the particle diffusion dominates) well and that is significantly better than the FLRW or FP on their own.

In our model, the field line random walk is facilitated by the turbulent interplanetary magnetic field, which is described by a power spectrum. A mix of 2D and slab modes in turbulent magnetic field gives rise to diffusive spreading of field lines (Matthaeus et al. 1995). This allows us to describe the path of a particle following a meandering field line using SDEs, with

$\mathrm{d} r_{\perp}\left(r_{\|}\right)=\sqrt{2 D_{\mathrm{FL}}\left(r_{\|}\right) \mathrm{d} r_{\|}} W_{\perp}$,

where $\mathrm{d} r_{\perp}$ is the displacement across the Parker field direction for advance $\mathrm{d} r_{\|}$along the field, $D_{\mathrm{FL}}$ is the field-line diffusion coefficient, and $W_{\perp}$ a Gaussian random number with zero mean and unit variance. The random-walking path is calculated for each simulated particle before the pseudo-particle trajectory is integrated. The pseudo-particles are propagated along and diffused from the meandering field lines according to Eq. (1), with crossfield diffusion given by Eq. (3), pitch angle diffusion as given in Eqs. (2) and (6), and focusing, where we used the Parker field geometry when calculating the focusing length.

The field-line diffusion coefficient was obtained as presented in Matthaeus et al. (1995), for composite slab and 2D turbulence. The contribution from the slab component is proportional to $S_{\|}\left(k_{\|}=0\right)$, which in our turbulence model (see below) vanishes. We therefore only considered the $2 \mathrm{D}$ contribution to the field-line diffusion, giving (Matthaeus et al. 1995)

$D_{\mathrm{FL}}=\left\{\frac{\int d^{2} \boldsymbol{k}_{\perp} S_{x x}\left(\boldsymbol{k}_{\perp}\right) / k_{\perp}^{2}}{B^{2}}\right\}^{1 / 2}$.
It should be noted that particle propagation as a beam along field lines that random-walk according to the field-line diffusion coefficient in Eq. (9) causes transport across the mean field much faster than diffusion with the coefficient given by Eq. (3). The latter aims to describe the cross-field spreading of particles at late times, when the pitch-angle distribution of the particles is isotropic. The two descriptions converge for a particle beam in turbulence with vanishing slab component, $a_{\mathrm{NLGC}}=1$ and a scatter-free velocity correlation $\left\langle v_{z}(0) v_{z}\left(t^{\prime}\right)\right\rangle=v_{z}^{2}$ in Eq. (3) of Matthaeus et al. (2003). The two descriptions of particle crossfield propagation are therefore consistent with each other in the appropriate limit.

Consistent with the SDE approach in the traditional model, the effect of the cross-field step length is not considered in the particle propagation time. This affects the accurate timing of particle propagation (e.g. Strauss \& Fichtner 2014).

\subsection{Turbulence model}

To compare the FP and FP+FLRW models, it is essential that $D_{\mu \mu}, \kappa_{\perp}$ and $D_{\mathrm{FL}}$, as defined in Eqs. (2), (3) and (9), are consistent with each other. We ensured this by deriving them for a simple interplanetary turbulence model. We considered only wave modes parallel (slab) and perpendicular (2D) to the mean magnetic field (Gray et al. 1996) and defined the power spectrum as

$S(\boldsymbol{k}) \equiv S\left(\boldsymbol{k}, r_{0}\right)=S_{\perp}\left(k_{\perp}\right) \delta\left(k_{\|}\right)+S_{\|}\left(k_{\|}\right) \delta\left(\boldsymbol{k}_{\perp}\right)$,

where $k_{\|}$and $k_{\perp}=\left|\boldsymbol{k}_{\perp}\right|$ are magnitudes of the wave number. The spectra are given as broken power laws

$S_{\|}\left(k_{\|}\right)= \begin{cases}C_{\|} L_{\|} \delta B_{\|}^{2} & L_{0}^{-1}<k_{\|}<L_{\|}^{-1} \\ \frac{C_{\|} L_{\|} \delta B_{\|}^{2}}{\left(L_{\|} k_{\|}\right)^{5 / 3}} & k_{\|} \geq L_{\|}^{-1}\end{cases}$

and

$2 \pi k_{\perp} S_{\perp}\left(k_{\perp}\right)= \begin{cases}C_{\perp} L_{\perp} \delta B_{\perp}^{2} & L_{0}^{-1}<k_{\perp}<L_{\perp}^{-1} \\ \frac{C_{\perp} L_{\perp} \delta B_{\perp}^{2}}{\left(L_{\perp} k_{\perp}\right)^{5 / 3}} & k_{\perp} \geq L_{\perp}^{-1},\end{cases}$

where $L_{\|}$and $L_{\perp}$ are the spectral breakpoint scales with a value of $0.007 \mathrm{AU}$ used for both scales, consistent with solar wind observations (e.g. Tu \& Marsch 1995, and references therein). The largest scale in the turbulence model, $L_{0}(r)$, is taken to be $r$, a natural choice for scaling in spherically expanding solar wind. The turbulence amplitude $\delta B^{2}$ is related to the normalisation factors $C_{\|}$and $C_{\perp}$ to give $\delta B_{\|, \perp}^{2}=2 \int S_{\|, \perp}(\boldsymbol{k}) \mathrm{d} \boldsymbol{k}$. The turbulence amplitudes are normalised to give the parallel scattering mean free path $\lambda_{\|}=0.3 \mathrm{AU}$ for a $10 \mathrm{MeV}$ proton at $1 \mathrm{AU}$ heliocentric distance (e.g. Palmer 1982), and $\delta B_{\|}^{2}: \delta B_{\perp}^{2}=20 \%: 80 \%$ (Bieber et al. 1996), giving $\delta B^{2} / B^{2}=0.04$ at $1 \mathrm{AU}$, in line with interplanetary turbulence observations (e.g. Burlaga \& Turner 1976).

The plasma turbulence evolves as the solar wind propagates from the Sun to the interplanetary medium (e.g. Tu \& Marsch 1995). The amplitude of the fluctuations changes as a function of radial distance as the properties of the background plasma change. The turbulence also evolves non-linearly, with energy 

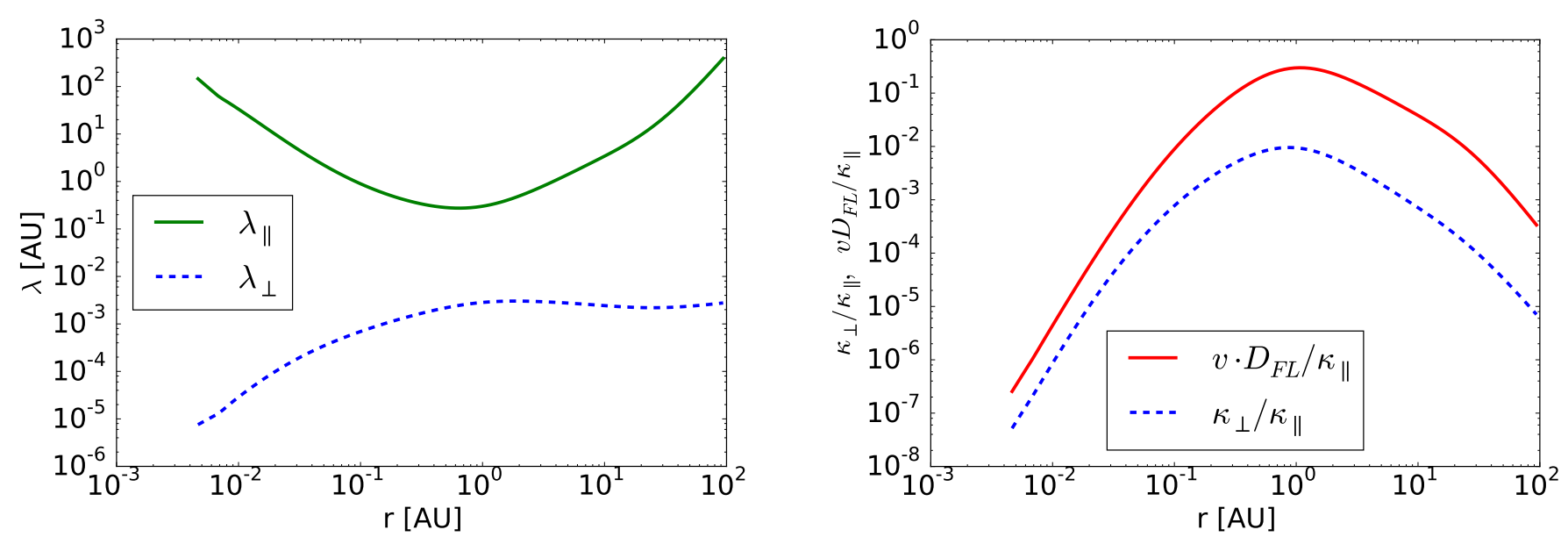

Fig. 1. Left: parallel and perpendicular scattering mean free paths for $10 \mathrm{MeV}$ protons obtained with Eqs. (2)-(4) and $\lambda_{\perp}=3 \kappa_{\perp} / v$. Right: diffusion coefficient ratios. For the red curve, the cross-field diffusion coefficient is obtained as $\kappa_{\perp}=v D_{\mathrm{FL}}$, representing a cross-field diffusion coefficient that is due to field-line meandering of unscattered particles.

cascading towards smaller scales. The radial and spectral evolution of turbulence in the heliosphere have been studied in several works over several decades. Many of the works, however, considered isotropic or slab turbulence (e.g. Tu et al. 1984; Tu 1987; Zhou \& Matthaeus 1990; Vainio et al. 2003) instead of the more realistic anisotropic geometry, or the composite mode used in this and many other recent SEP transport studies. Some formulations exist for spectral transport separately to small parallel and perpendicular scales (e.g. Cranmer \& van Ballegooijen 2005; Laitinen 2005). These works, however, have not been considered with respect to particle transport coefficients. The recent detailed heliospheric turbulence transport model by Zank et al. (2012) provides the evolution of the total turbulence energy and correlation lengths instead of the turbulence spectrum, and the application of their model to SEP transport modelling is a challenging task that has so far not been attempted.

Developing an SEP transport model that considers the spectral evolution of composite turbulence in the heliosphere is therefore an undertaking that has not been considered before, and it warrants a separate study. In this work, we take a simpler approach and only consider the radial evolution of the turbulence. We used the WKB description (Richter \& Olbers 1974; Tu et al. 1984) for the slab and 2D components. For simplicity, we did not consider the evolution of the spectral shape nor the wave refraction, and we kept the wave components in their pure slab and $2 \mathrm{D}$ geometries. Furthermore, we did not consider the change in $k$ that is due to the varying wave phase speed because assuming a constant inertial-frame wave phase speed agrees well with observations (Vainio et al. 2003). With these simplifications, the turbulence evolves as

$$
\begin{aligned}
S_{\|, \perp}\left(k_{\|, \perp}, r\right) & =S_{\|, \perp}\left(k_{\|, \perp}, r_{0}\right)\left[\frac{V_{r 0} v_{\mathrm{Ar}}}{V_{r} v_{\mathrm{Ar} 0}}\right]^{2}\left(\frac{n_{\mathrm{e}}}{n_{e 0}}\right)^{1 / 2} \\
& \equiv S_{\|, \perp}\left(k_{\|, \perp}, r_{0}\right) W(r),
\end{aligned}
$$

where $v_{\mathrm{Ar}}$ is the radial component of Alfvén velocity, $V_{r}=$ $V_{\mathrm{sw}, r}+v_{\mathrm{Ar}}$ and $n_{\mathrm{e}}$ the solar wind electron number density, with the subscript 0 denoting the values at reference distance $r_{0}$. We used a constant solar wind velocity $V_{\mathrm{sw}, r 0}$ and electron density $n_{\mathrm{e}}(r)=n_{\mathrm{e} 0} r_{0}^{2} / r^{2}$. Using these, we find

$W(r)=\left(\frac{r_{0}}{r}\right)^{3}\left(\frac{V_{\mathrm{sw}, r 0}+v_{\mathrm{A} 0}}{V_{\mathrm{sw}, r 0}+\frac{r_{0}}{r} v_{\mathrm{A} 0}}\right)^{2}$, which is consistent with the observed approximately $\propto r^{-3}$ trend in interplanetary space (e.g. Bavassano et al. 1982). We here used $V_{\mathrm{sw}, r 0}=400 \mathrm{~km} \mathrm{~s}^{-1}$ and $v_{\mathrm{a}, r 0}=30 \mathrm{~km} \mathrm{~s}^{-1}$ to represent the values at $r_{0}=1 \mathrm{AU}$.

It should be noted that our model for the field-line random walk differs from that of Giacalone (2001), where the field-line random walk is caused by the motion of the magnetic field footpoints at the Sun that are due to supergranulation. The Giacalone (2001) model provides an explanation for the source of the fieldline random walk at the Sun, but it does not allow for turbulence evolution in interplanetary space, which further modifies the turbulence as the magnetic fields are convected with the solar wind (e.g. Bruno \& Carbone 2005, and references therein). In our model, the field-line meandering is derived from the turbulence spectrum and the corresponding field line diffusion coefficient, with no limiting assumptions on the interplanetary evolution of the turbulence.

\subsection{Transport parameters}

After specifying a turbulence model, we now calculate the particle transport parameters that are fully consistent with the properties of the turbulence for both the FP and FP+FLRW models. The transport parameters are shown in Fig. 1 for $10 \mathrm{MeV}$ protons. In the left panel we show the particle transport parameters as parallel and cross-field mean free paths, which are the same for both the FP and the FP+FLRW model, and the right panel shows the ratio of the cross-field and parallel diffusion coefficients, $\kappa_{\perp} / \kappa_{\|}$. The values of the particle diffusion coefficients are broadly similar to those used in other studies (e.g. Zhang et al. 2009; Pei et al. 2010).

At small heliocentric distances, our model gives high values for the parallel mean free path (left panel of Fig. 1), allowing the particles to propagate along the field lines essentially without scattering from the Sun to 0.4 AU. A similar transport regime is also present in models that use a constant radial diffusion coefficient to parametrise the parallel diffusion coefficient (e.g. Zhang et al. 2009; Dröge et al. 2010; He et al. 2011; Giacalone \& Jokipii 2012; Qin et al. 2013).

In addition to $\kappa_{\perp} / \kappa_{\|}$, we show also the ratio for particles that propagate as a beam along the meandering field line, $v \cdot D_{\mathrm{FL}}$, in the right panel of Fig. 1. As discussed in Sect. 2.2, $v \cdot D_{\mathrm{FL}}$ is always larger than $\kappa_{\perp}$. In addition, as shown by 
T. Laitinen et al.: Solar energetic particle access to distant longitudes through turbulent field-line meandering
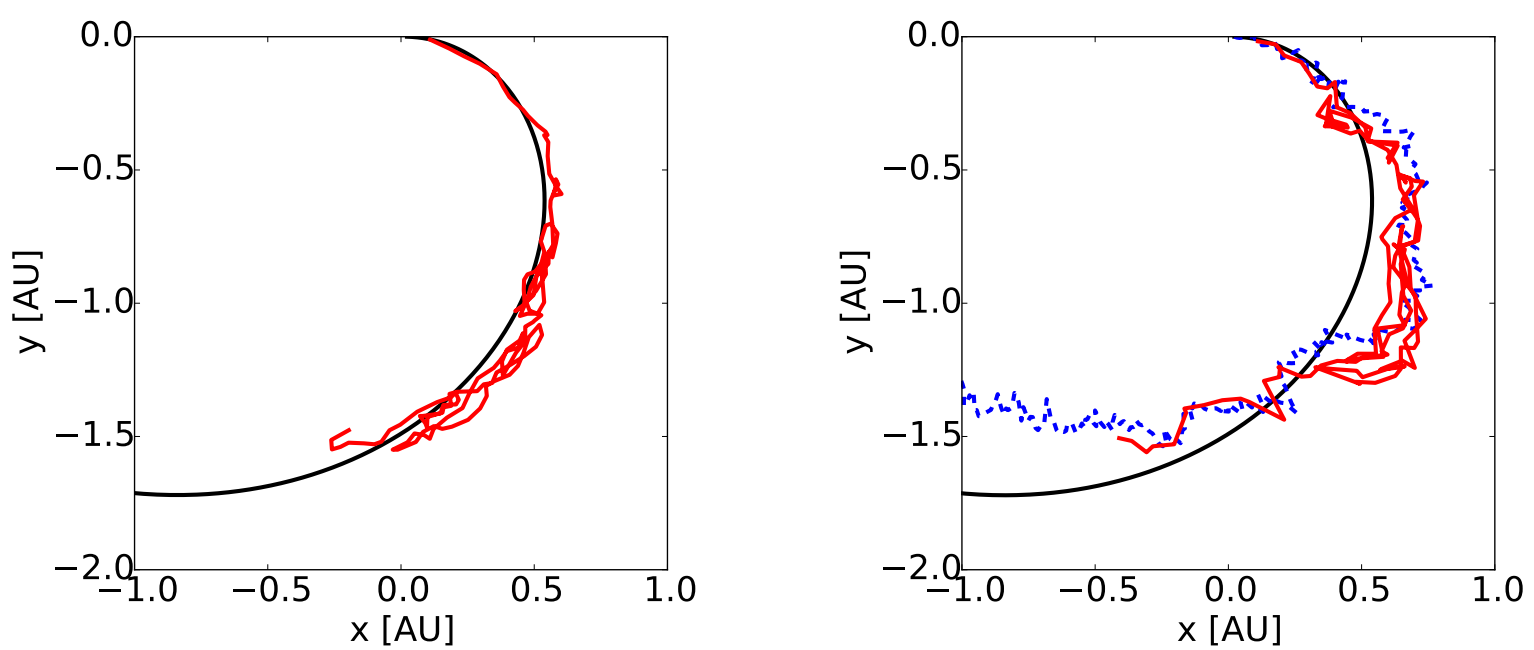

Fig. 2. Sample path (red curve) of a $10 \mathrm{MeV}$ proton injected at the Sun (located at origin) with the FP (left panel) and FP+FLRW model (right panel), respectively. The black curve shows the Parker spiral field, while the dashed blue curve shows a meandering field line in the FP+FLRW model (right panel).

Fraschetti \& Jokipii (2011) and Fraschetti \& Giacalone (2012), for typical interplanetary turbulence parameters the rate of decoupling of particles from their field lines is always slower than the spreading of particles across the mean field. Thus, at early times the SEP propagation along random-walking field lines dominates over both the asymptotic perpendicular diffusion and the particle decoupling from the field.

The cross-field mean free path for both particles and field lines is very small close to the Sun, of the order of $10^{-5}$ AU. The value is deceptive, however: a low value close to the Sun can result in large longitudinal spread. In radial geometry, the fieldline diffusion coefficient can be written in terms of a longitudinal diffusion coefficient, $D_{\mathrm{FL}}=\left\langle\Delta x^{2}\right\rangle /(2 r)=r^{2}\left\langle\Delta \phi^{2}\right\rangle /(2 r) \equiv$ $r^{2} D_{\mathrm{FL} \phi}$. In a radial geometry, a constant longitudinal diffusion coefficient is therefore represented by a diffusion coefficient that is proportional to $r^{2}$. For our turbulence model, we have $D_{\mathrm{FL} \phi}=\left(40^{\circ}\right)^{2} / \mathrm{AU}$ close to the Sun, and $D_{\mathrm{FL} \phi}=\left(10^{\circ}\right)^{2} / \mathrm{AU}$ at $1 \mathrm{AU}$.

It should be noted that a simple parametrisation with $\kappa_{\perp} / \kappa_{\|} \mathrm{a}$ constant or $\propto r$ does not reproduce the behaviour of the diffusion coefficients obtained with a full model of the turbulence, such as the one used in this study and in Pei et al. (2010). The radial evolution of turbulence and its effect on the diffusion coefficients will be discussed in more detail in a separate study.

\section{Results and discussion}

We here study a simple injection profile,

$$
\begin{aligned}
Q(r, \theta, \phi, t)= & \delta\left(r-1 \mathrm{r}_{\odot}\right) \delta(\theta-\pi / 2) \\
& \times \delta(\mu-1) \delta\left(E-E_{0}\right) \delta(\phi) \delta\left(t-t_{0}\right),
\end{aligned}
$$

for $E_{0}=10 \mathrm{MeV}$ protons and trace the propagation of pseudoparticles through interplanetary space according to the model equations. The resulting profile can also be convolved with different injection profiles to produce response for time- and longitude-extended injections, which will be a subject of a future full parameter study.

The propagation of the particles in FP and FP+FLRW models is shown in Fig. 2 as a 2D projection of the 3D orbits. The red curves depict the path of a simulated pseudo-particle, and the black curves show the Parker spiral. In the right panel, the blue dashed curve depicts the diffusively meandering field line. In both models, the particles scatter along the field line and diffuse away from it in the cross-field direction. In the FP+FLRW model, however, the field line itself meanders away from the Parker Spiral field. As discussed above, the diffusion of the field line is faster than the diffusion of a particle across the field line, thus the particle orbit tends to follow the meandering field line (blue dashed curve in Fig. 2), and as a result particles spread faster across the mean Parker spiral field than in the FP case.

In Fig. 3 we compare the particle distributions predicted by the diffusion (FP) (left) and our new FP+FLRW (right) model, one (top row) and three (bottom row) hours after injection. The distribution contours are calculated for particles near the ecliptic plane, between latitudes $\pm 10^{\circ}$. In the FP model, particles diffusively spread in longitude as a function of time, and as they are focused rapidly away from the Sun in the diverging magnetic field, the most significant spreading of the particles takes place at distances $>0.5 \mathrm{AU}$. In the FP+FLRW model, however, the particles have a much wider distribution in longitude and show significant spreading close to the Sun.

To compare the models with observed SEP events, we show in Fig. 4 the time evolution of the simulated intensities at three different longitudes for the two models, compared to an event that took place during November 3-4, 2011. Gómez-Herrero et al. (2015) have analysed this event using a 1D transport model, which, based on anisotropy observations, suggests a source region with $270^{\circ}$ longitudinal extent. However, they found no supporting evidence for such a wide source and suggested a disturbed interplanetary magnetic field as one of the possible causes for the event extent.

The SEP event is related to a solar eruption at longitude $50^{\circ}$ east of the STEREO B (STB) spacecraft, with an M4.7 flare maximum at $t_{\max }=22: 41 \mathrm{UT}$ on November 3 at N20E62 from Earth's point of view (Richardson et al. 2014; Gómez-Herrero et al. 2015). Using the longitude angle $\phi$ as defined in Fig. 3, where the particle source is at $\phi=0^{\circ}$, STB was at longitude $\phi_{\mathrm{STB}}=50^{\circ}$, (see lower right panel of Fig. 4). In this coordinate system, the SEP source is connected along the Parker field line (solid black spiral curve in the lower right panel of 

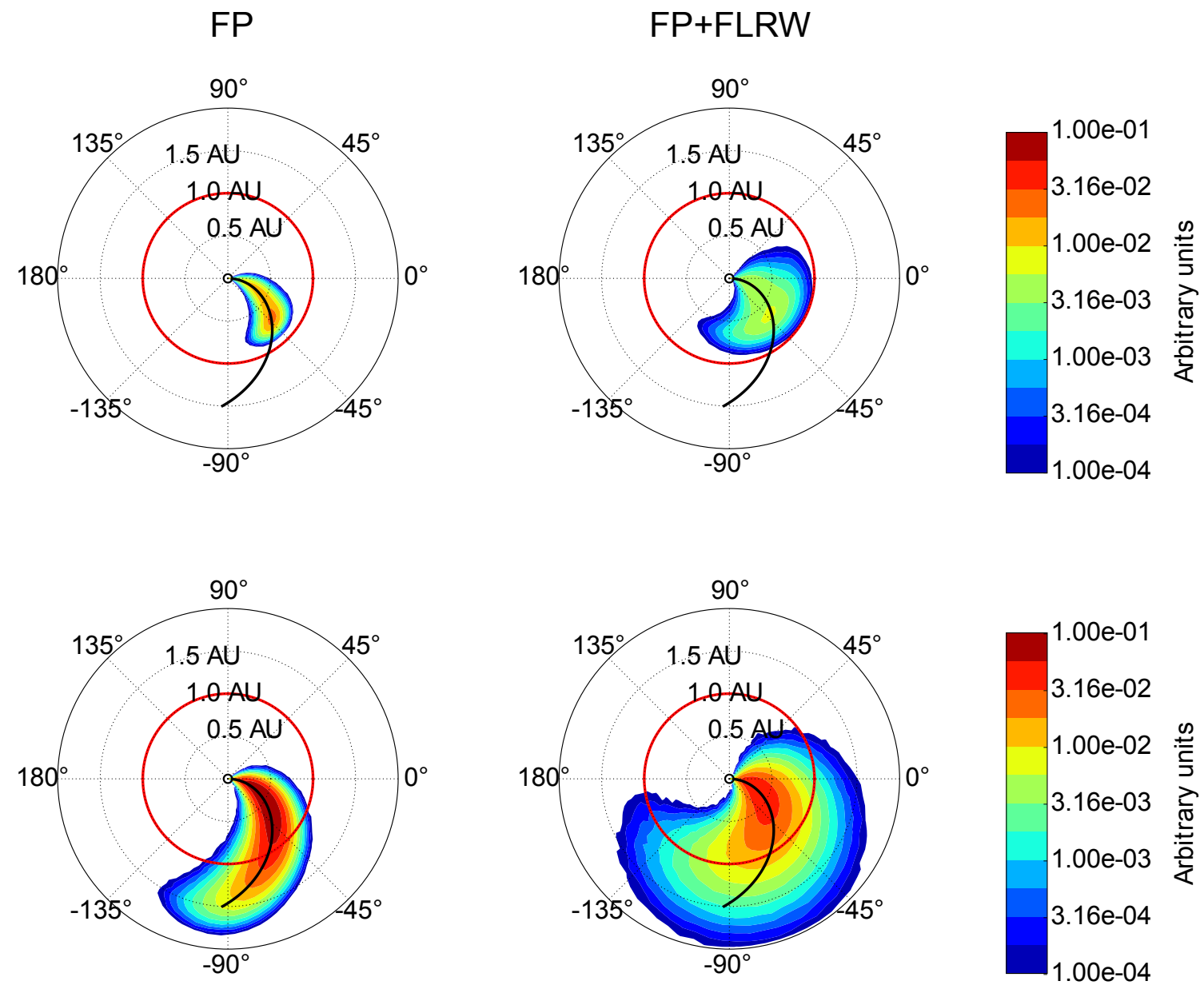

Fig. 3. SEP particle density in arbitrary units for particles between latitudes $-10^{\circ}$ and $10^{\circ}$, one hour (top row) and three hours (bottom row) after an impulsive injection at 1 solar radius and longitude $\phi=0^{\circ}$. Left panels show the result of the FP model, while the right panels show the FP+FLRW model. The black curve shows the Parker field-line starting at $\phi=0^{\circ}$. The field line reaches a radial distance of $1 \mathrm{AU}$ (red circle) at $\phi=-62^{\circ}$.

Fig. 4) to longitude $\phi=-62^{\circ}$ at radial distance of $1 \mathrm{AU}$ (red circle). During the event, the STEREO A (STA) and SOHO spacecraft were located at longitudes $\phi_{\mathrm{STA}}=-103^{\circ}$ and $\phi_{\mathrm{SOHO}}=$ $152^{\circ}$, respectively.

Observed intensities of $6-10 \mathrm{MeV}$ protons are shown in Fig. 4 with dashed green curves. They were obtained with ERNE onboard SOHO (Torsti et al. 1995) (top left panel), LET onboard STB (Mewaldt et al. 2008) (top right), and the LET instrument onboard STA (bottom left). The blue and red curves in Fig. 4 show the intensities from the FP and FP+FLRW models, respectively, for the injection function $Q$ with $t_{0}=t_{\max }-500 \mathrm{~s}$, (subtracting $500 \mathrm{~s}$ of light-travelling time from Sun to $1 \mathrm{AU}$ ). The injection intensity is fitted so that the highest intensities of the models match the observed SEP highest intensity at STA.

The SEP event was best observed with LET/STA, located $41^{\circ}$ clockwise from the well-connected longitude of $\phi=-62^{\circ}$. The $6-10 \mathrm{MeV}$ proton intensity increased by 5 orders of magnitude within a few hours (bottom left panel of Fig. 4). The event was also observed by LET/STB and ERNE/SOHO at $112^{\circ}$ and $146^{\circ}$, respectively, from the well-connected field-line. Even with such poor connection, SEP intensities began to rise within an hour of the observed SEP onset at STA, to rise by 3 orders of magnitude above the pre-event background. It is therefore clear that the SEPs had rapid access to a wide range of longitudes during this solar event.
While we did not perform a full transport fitting in this report, our study implies that the rapid access of SEPs during the November 3-4, 2011 event to the whole inner heliosphere can be achieved with our FP+FLRW model even with a narrow source region. The FP+FLRW-modelled particles are observed within an hour at the longitudes corresponding to the November 3, 2011 spacecraft locations. The FP model can reproduce the rapid onset of the SEP event at the STA spacecraft, with a delay of half an hour compared to the FP+FLRW model. It is not capable of reproducing the SOHO or STB observations, however, because it fails to spread particles to these longitudes to intensity levels above the pre-event background during the first ten hours of the event.

The inability of the FP model to spread particles to the longitudes of the SOHO and STB spacecraft within the first ten hours is shown in Fig. 5, where we show the evolution of the simulated SEP event as it would have been measured at $1 \mathrm{AU}$ at different heliographic longitudes, with $t_{0}=0$. For the FP model the intensity falls very sharply as a function of longitude, explaining the failure of the FP model in Fig. 4 to describe the November 2011 event without resorting to extremely wide source regions (Gómez-Herrero et al. 2015). As shown in Fig. 5, the SEPs spread quickly for the FP+FLRW model, and the SEP onset within a wide range of longitudes, between $\phi=-150^{\circ}$ and $50^{\circ}$, is delayed only by an hour compared to the onset at the wellconnected longitude, $\phi=-62^{\circ}$. Such a rapid spreading of first 
T. Laitinen et al.: Solar energetic particle access to distant longitudes through turbulent field-line meandering
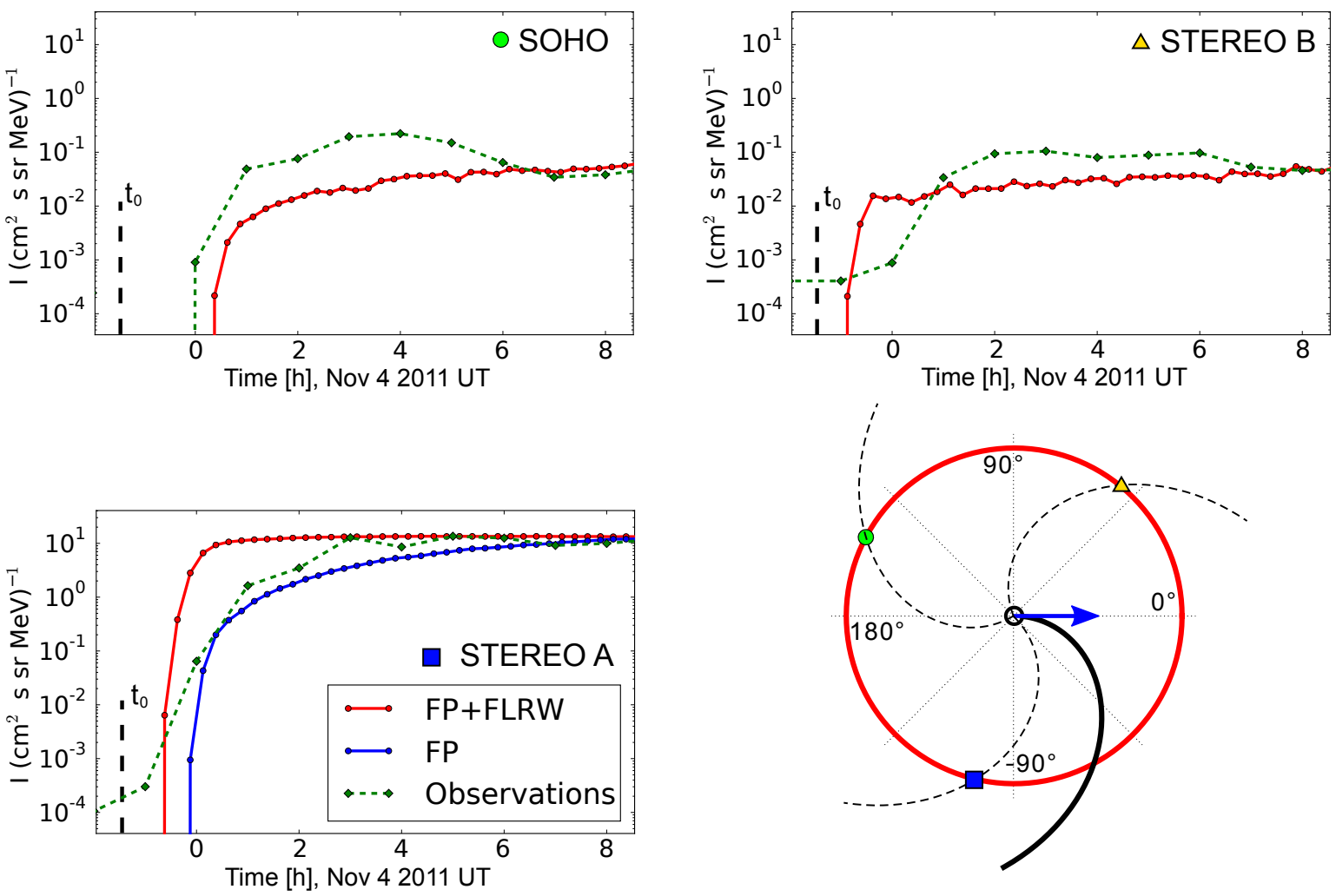

Fig. 4. SEP proton intensities as a function of time at $1 \mathrm{AU}$. The green dashed curve shows 6-10 MeV SEP proton intensities observed during a solar eruption on Nov. 3-4, 2011, at SOHO (top left panel), STEREO B (top right) and STEREO A (bottom left) spacecraft. The blue and red curves show the $10 \mathrm{MeV}$ proton intensities simulated using the FP and FP+FLRW models, respectively, using an impulsive injection at flare maximum, $t_{0}=22: 41-500 \mathrm{~s}$ UT on Nov. 3 (dashed vertical line) at the flare longitude. The spacecraft locations are shown in the bottom right panel by filled green circle, yellow triangle, and blue square for SOHO, STEREO B, and STEREO A, respectively. The blue arrow denotes the SEP injection longitude, the thick solid line and red circle are the same as in Fig. 3, and the dashed curves show the Parker spiral field-lines connecting the spacecraft to the solar surface.

particles is a key feature of observations: the earliest SEP onsets at $100^{\circ}$ from the flare longitude are delayed only an hour compared to onsets at best-connected longitude (Richardson et al. 2014).

The longitudinal extent of an SEP event is frequently described by means of a Gaussian fit, $f(\phi)=f_{0} \exp \{-(\phi-$ $\left.\left.\phi_{0}\right)^{2} /\left(2 \sigma^{2}\right)\right\}$, to the peak SEP intensities observed by spacecraft at different longitudes. Using STEREO and near-Earth spacecraft, several studies have reported values of $\sigma=30^{\circ}-50^{\circ}$ to best represent the range of SEP event widths (Lario et al. 2006, 2013; Wiedenbeck et al. 2013; Dresing et al. 2014; Richardson et al. 2014). To compare our models to observations, we performed a Gaussian fit to the peak intensities of the simulated SEP events at $1 \mathrm{AU}$ for the two models presented in this work. The longitudinal distributions and the corresponding fits are shown in Fig. 6 with solid and dashed curves, respectively. The grey area depicts the observational range of $\sigma=30^{\circ}-50^{\circ}$. The FP+FLRW model (red curve) can reproduce the observed SEP event widths, with width $\sigma=34^{\circ}$, while the FP model (blue curve) can not; it has a very narrow SEP width, with only $\sigma=11^{\circ}$. This strong difference between the FP+FLRW and FP models is consistent with Laitinen et al. (2013), who found for Cartesian geometry that at early times the mean square width in full-orbit simulations exceeded the FP model result by an order of magnitude and that an FP+FLRW model reproduces the behaviour seen in full-orbit simulations well.

Our results show the importance of modelling the early phase of an SEP event using a proper physical description. Both the FP
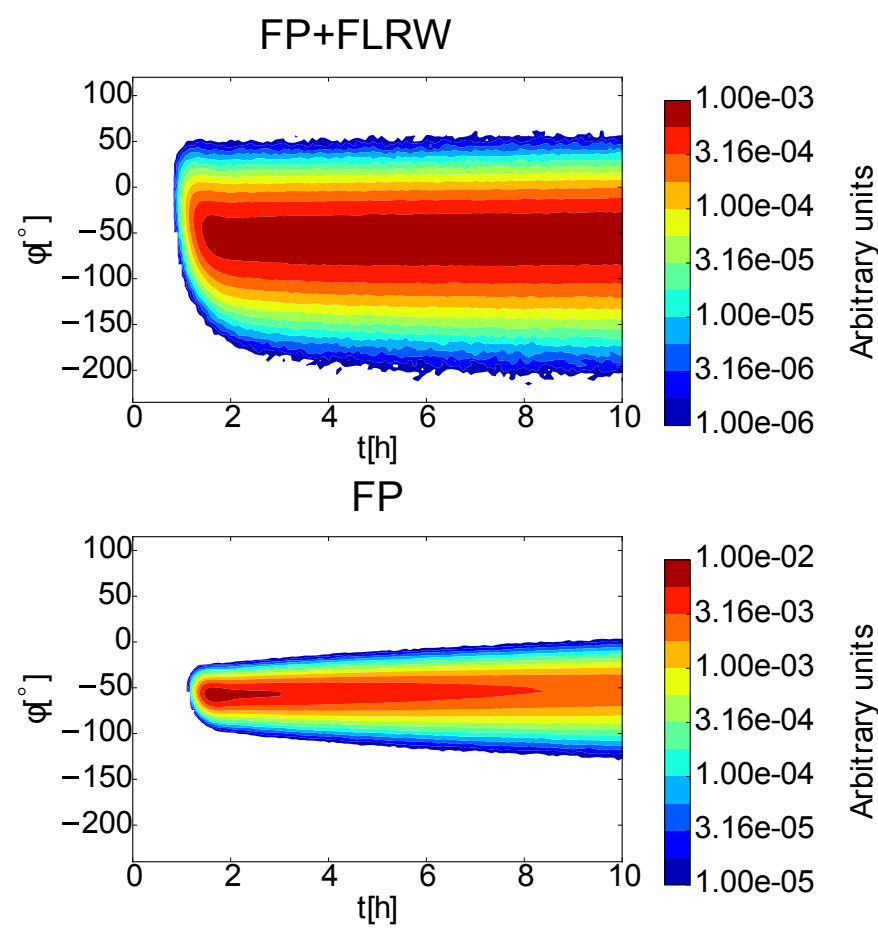

Fig. 5. Contour plot of particle density in arbitrary units as a function of time and longitude at $1 \mathrm{AU}$ radial distance from the Sun (red circle in Figs. 1 and 2). The top panel shows the FP+FLRW model result, while the bottom panel shows results for the FP model. 


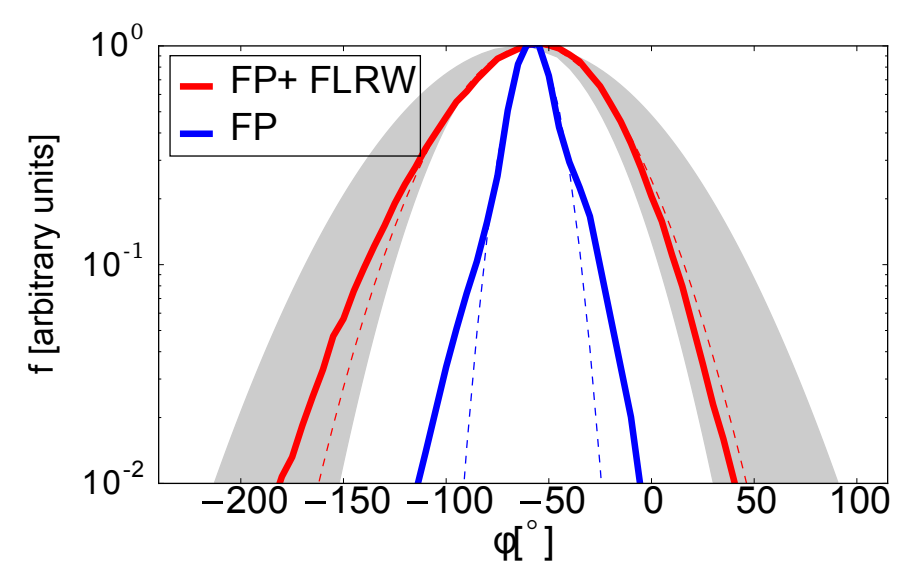

Fig. 6. Peak intensity at $1 \mathrm{AU}$ as a function of longitude for the FP (blue solid curve) and FP+FLRW (red solid curve) models. The dashed lines show the fits of Gaussian profiles to the peak intensities, with $\sigma=$ $11^{\circ}$ for the FP model, and $\sigma=34^{\circ}$ for the FP+FLRW model. The grey area depicts the range of Gaussian widths of $30-50^{\circ}$ obtained from observations.

and FP+FLRW models aim to describe the effect of the turbulence on the particles, including the cross-field spreading that is due to field-line meandering. In the FP model, the field-line meandering is included only time-asymptotically using a diffusion description. However, as shown by Laitinen et al. (2013), the initial spreading of particles is fast and non-diffusive, relaxing to diffusive behaviour only at later times. This is accounted for in our new FP+FLRW model and results in particles reaching much broader longitudinal extents than predicted by an FP model using the same turbulence parameters.

Propagation along meandering field lines implies that similar event extents should also be reached with other particle species. This is indeed found in observations: Lario et al. (2013) and Richardson et al. (2014) have found a similar range of peak intensity widths for electrons and protons at different energies, and Wiedenbeck et al. (2013) and Cohen et al. (2014) for heavy ions during impulsive events. While these observations do not exclude other SEP-spreading mechanisms, such as transport in the corona, symphatetic flaring and extended particle sources (see, e.g., Wiedenbeck et al. 2013; Gómez-Herrero et al. 2015), the effect of interplanetary field-line meandering offers a direct explanation for the SEP event widths that are observed to be independent of energy and particle species and the gradual or impulsive classification. The influence of the source height, size, and different interplanetary conditions on the resulting SEP event extent will be explored in a future full parametric study. We will also address the varying degrees of observed SEP anisotropies, which typically indicate cross-field transport in the interplanetary space (Dresing et al. 2014), but in some cases indicate rapid access of particles throughout the heliosphere (Gómez-Herrero et al. 2015).

\section{Conclusions}

We developed a new SEP transport model that takes the nondiffusive propagation of SEPs early in the event history into account for a Parker spiral geometry. We showed that the early onset of SEPs over a wide range of longitudes can be explained by field-line random walk and requires an SEP transport model that properly describes the non-diffusive early phase of SEP crossfield propagation. Our FP+FLRW model is the first model that is capable of reproducing the observed fast access of SEPs to distant longitudes, when the particle and field-line diffusion coefficients are consistently derived from an interplanetary turbulence model. When the FLRW is not included (in the FP model), a much narrower cross-field extent of the SEP event is produced. We conclude that introducing field-line wandering into SEP modelling has the potential of resolving the problem of fast access of SEPs to a wide range of longitudes.

Acknowledgements. We acknowledge the STEREO/IMPACT team for providing the data used in this study. The SOHO/ERNE data were obtained from the Space Research Laboratory, University of Turku (http://www.srl.utu.fi/erne_ data/). T.L. and S.D. acknowledge support from the UK Science and Technology Facilities Council (STFC) (grants ST/J001341/1 and ST/M00760X/1), M.M. from the European Commission FP7 Project COMESEP (263252), and F.E. from NASA grant NNX14AG03G. The contribution of A.K. benefitted from financial support through project He 3279/15-1, funded by the Deutsche Forschungsgmeinschaft (DFG), at the CAU Kiel, where large parts of this work where carried out. T.L., F.E. and S.D. acknowledge support from the International Space Science Institute as part of international team 297. We thank Horst Fichtner for helpful discussions. Access to the University of Central Lancashire's High Performance Computing Facility is gratefully acknowledged.

\section{References}

Bavassano, B., Dobrowolny, M., Mariani, F., \& Ness, N. F. 1982, J. Geophys. Res., 87, 3617

Beeck, J., \& Wibberenz, G. 1986, ApJ, 311, 437

Bieber, J. W., Wanner, W., \& Matthaeus, W. H. 1996, J. Geophys. Res., 101, 2511

Bruno, R., \& Carbone, V. 2005, Liv. Rev. Sol. Phys., 2, 4

Burger, R. A., Potgieter, M. S., \& Heber, B. 2000, J. Geophys. Res., 105, 27447 Burlaga, L. F., \& Turner, J. M. 1976, J. Geophys. Res., 81, 73

Cohen, C. M. S., Mason, G. M., Mewaldt, R. A., \& Wiedenbeck, M. E. 2014, ApJ, 793, 35

Cranmer, S. R., \& van Ballegooijen, A. A. 2005, ApJS, 156, 265

Dalla, S., Marsh, M. S., \& Laitinen, T. 2015, ApJ, 808, 62

Dresing, N., Gómez-Herrero, R., Klassen, A., et al. 2012, Sol. Phys., 281, 281

Dresing, N., Gómez-Herrero, R., Heber, B., et al. 2014, A\&A, 567, A27

Dröge, W., Kartavykh, Y. Y., Klecker, B., \& Kovaltsov, G. A. 2010, ApJ, 709, 912

Dröge, W., Kartavykh, Y. Y., Dresing, N., Heber, B., \& Klassen, A. 2014, J. Geophys. Res., 119, 6074

Dwyer, J. R., Mason, G. M., Mazur, J. E., et al. 1997, ApJ, 490, L115

Effenberger, F., Fichtner, H., Scherer, K., et al. 2012, ApJ, 750, 108

Fraschetti, F., \& Giacalone, J. 2012, ApJ, 755, 114

Fraschetti, F., \& Jokipii, J. R. 2011, ApJ, 734, 83

Gardiner, C. W. 2009, in Stochastic Methods, 4th edn. (Berlin, Heidelberg: Springer-Verlag), 13

Giacalone, J. 2001, J. Geophys. Res., 106, 15881

Giacalone, J., \& Jokipii, J. R. 1999, ApJ, 520, 204

Giacalone, J., \& Jokipii, J. R. 2012, ApJ, 751, L33

Giacalone, J., Jokipii, J. R., \& Mazur, J. E. 2000, ApJ, 532, L75

Gómez-Herrero, R., Dresing, N., Klassen, A., et al. 2015, ApJ, 799, 55

Gray, P. C., Pontius, D. H., \& Matthaeus, W. H. 1996, Geophys. Res. Lett., 23, 965

He, H.-Q., Qin, G., \& Zhang, M. 2011, ApJ, 734, 74

Isenberg, P. A. 1997, J. Geophys. Res., 102, 4719

Jokipii, J. R. 1966, ApJ, 146, 480

Kelly, J., Dalla, S., \& Laitinen, T. 2012, ApJ, 750, 47

Kopp, A., Büsching, I., Strauss, R. D., \& Potgieter, M. S. 2012, Comput. Phys. Comm., 183, 530

Kóta, J., \& Jokipii, J. R. 2000, ApJ, 531, 1067

Laitinen, T. 2005, Astrophys. Space Sci. Trans., 1, 35

Laitinen, T., Dalla, S., \& Marsh, M. S. 2013, ApJ, 773, L29

Lario, D., Kallenrode, M.-B., Decker, R. B., et al. 2006, ApJ, 653, 1531

Lario, D., Aran, A., Gómez-Herrero, R., et al. 2013, ApJ, 767, 41

le Roux, J. A., \& Webb, G. M. 2009, ApJ, 693, 534

Marsh, M. S., Dalla, S., Kelly, J., \& Laitinen, T. 2013, ApJ, 774, 4

Matthaeus, W. H., Gray, P. C., Pontius, Jr., D. H., \& Bieber, J. W. 1995, Phys. Rev. Lett., 75, 2136

Matthaeus, W. H., Qin, G., Bieber, J. W., \& Zank, G. P. 2003, ApJ, 590, L53 Mewaldt, R. A., Cohen, C. M. S., Cook, W. R., et al. 2008, Space Sci. Rev., 136, 285

Palmer, I. D. 1982, Rev. Geophys. Space Phys., 20, 335

Parker, E. N. 1965, Planet. Space Sci., 13, 9 
Pei, C., Bieber, J. W., Burger, R. A., \& Clem, J. 2010, J. Geophys. Res., 115, 12107

Potgieter, M. S., Vos, E. E., Boezio, M., et al. 2014, Sol. Phys., 289, 391

Qin, G., \& Shalchi, A. 2014, Appl. Phys. Res., 6, 1

Qin, G., Matthaeus, W. H., \& Bieber, J. W. 2002, ApJ, 578, L117

Qin, G., Wang, Y., Zhang, M., \& Dalla, S. 2013, ApJ, 766, 74

Reames, D. V. 1999, Space Sci. Rev., 90, 413

Richardson, I. G., von Rosenvinge, T. T., Cane, H. V., et al. 2014, Sol. Phys., 289, 3059

Richter, A. K., \& Olbers, D. J. 1974, Astrophys. Space Sci., 26, 95

Roelof, E. C. 1969, in Lectures in High-Energy Astrophysics, eds. H. Ögelman, \& J. R. Wayland, 111

Ruffolo, D. 1995, ApJ, 442, 861

Shalchi, A. 2010, ApJ, 720, L127

Skilling, J. 1971, ApJ, 170, 265

Strauss, R. D., \& Fichtner, H. 2014, A\&A, 572, L3

Strauss, R. D., \& Fichtner, H. 2015, ApJ, 801, 29

Torsti, J., Valtonen, E., Lumme, M., et al. 1995, Sol. Phys., 162, 505

Tu, C.-Y. 1987, Sol. Phys., 109, 149

Tu, C.-Y., \& Marsch, E. 1995, Space Sci. Rev., 73, 1

Tu, C.-Y., Pu, Z.-Y., \& Wei, F.-S. 1984, J. Geophys. Res., 89, 9695

Vainio, R., Laitinen, T., \& Fichtner, H. 2003, A\&A, 407, 713

Webb, G. M., Le Roux, J. A., \& Zank, G. P. 2009, in AIP Conf. Ser. 1183, eds

X. Ao, \& G. Z. R. Burrows, 85

Wiedenbeck, M. E., Mason, G. M., Cohen, C. M. S., et al. 2013, ApJ, 762, 54

Zank, G. P., Dosch, A., Hunana, P., et al. 2012, ApJ, 745, 35

Zhang, M., Jokipii, J. R., \& McKibben, R. B. 2003, ApJ, 595, 493

Zhang, M., Qin, G., \& Rassoul, H. 2009, ApJ, 692, 109

Zhou, Y., \& Matthaeus, W. H. 1990, J. Geophys. Res., 95, 10291

\section{Appendix A: Stochastic differential equations in the FP and FP+FLRW models}

The method of stochastic differential equations (SDEs) is a tool for solving Fokker-Planck-type transport equations. Instead of solving this equation directly, as is the case when applying finite-difference methods, for example, the SDEs trace socalled pseudo-particles, that is, phase-space elements, through the phase-space. These pseudo-particles obey the corresponding Langevin equation. The simplest example of a transport equation is

$\frac{\partial f}{\partial t}=-v \frac{\partial f}{\partial x}+D \frac{\partial^{2} f}{\partial x^{2}}$

with the Langevin equation

$\mathrm{d} x=v \mathrm{~d} t+\sqrt{2 D \mathrm{~d} t} W_{t}$,

where $f(x, t)$ is the distribution function that depends on space $x$ and time $t . v$ and $D$ denote velocity and diffusion coefficient, $\mathrm{d} t$ is the time step and and $W_{t}$ a Gaussian random number with zero mean and unit variance. The solution of the entire equation is obtained by binning the results for an appropriate number of pseudo-particles. Although these phase-space elements represent the behaviour of real particles in most cases quite well, they should not be confused with the latter. In contrast to the traditional approach, the SDE method also allows solving the transport equation, with a different Langevin equation, backwards in time. For details on these points and on the method in general, we refer to Kopp et al. (2012) and the literature cited there.

In the present case we solved the transport equation, Eq. (1), under the assumption of a pure radial and constant solar wind $\left(\boldsymbol{V}_{\mathrm{sw}}=V_{\mathrm{sw}} \boldsymbol{e}_{r}\right)$ and a vanishing latitudinal component of the magnetic field, which in particular significantly reduces the Skilling term $R$ (the term in curved brackets in the second line of Eq. (1)). We first solved the equation in a local Cartesian system with $z$ being the direction along the magnetic field, and transformed the transport quantities back to the global system before the actual integration was carried out (Effenberger et al. 2012). In this local system, the Langevin equations read

$$
\begin{aligned}
& \mathrm{d} x=\left(\boldsymbol{u}_{\perp, x}+\frac{\partial \kappa_{\perp, x} \mathrm{~d} t}{\partial x}\right) \mathrm{d} t+\sqrt{2 \kappa_{\perp, x}} W_{t, x}, \\
& \mathrm{~d} y=\left(\boldsymbol{u}_{\perp, y}+\frac{\partial \kappa_{\perp, y} \mathrm{~d} t}{\partial y}\right) \mathrm{d} t+\sqrt{2 \kappa_{\perp, y}} W_{t, y}, \\
& \mathrm{~d} \mu=\frac{1}{2}\left(\frac{v}{L}+\mu R\right)\left(1-\mu^{2}\right) \mathrm{d} t+\frac{\partial D_{\mu \mu}}{\partial \mu} \mathrm{d} t+\sqrt{2 D_{\mu \mu} \mathrm{d} t} W_{t, \mu} .
\end{aligned}
$$

Here, $u_{\|}=V_{\mathrm{sw}} b_{r}$, where $b_{r}$ is the radial component of the unit vector $\hat{\boldsymbol{b}}=\mathrm{e}_{z}$ along the local magnetic field line and $\boldsymbol{u}_{\perp}=V_{\mathrm{sw}}\left(\boldsymbol{e}_{r}-b_{r} \hat{\boldsymbol{b}}\right)$. The two local unit vectors perpendicular to the magnetic field are $\boldsymbol{e}_{x}=\boldsymbol{e}_{\theta} \times \boldsymbol{e}_{z}$ and $\boldsymbol{e}_{y}=\boldsymbol{e}_{z} \times \boldsymbol{e}_{x}$, and the corresponding particle cross-field diffusion coefficients $\kappa_{\perp, x}$ and $\kappa_{\perp, y}$, respectively. For the FP model, $\hat{b}$ is the unit vector along the local Parker field direction, whereas for the FP+FLRW model the pseudo-particle is first propagated (virtually) along the meandering field line, and $\hat{b}$ itself is computed through the difference between the old and new points.

The transport coefficients in Eq. (A.3) are transformed into the global system by means of the tensor $T_{\alpha \beta}=e_{\alpha, \beta}$, so that the (spatial) diffusion tensor becomes non-diagonal. In this case, the square root of a tensor has to be computed, see Kopp et al. (2012).

It is important to note that calculating the meandering field line using Eq. (8) is performed before the particle is injected into the meandering field line. The particle cross-field propagation, while diffusive relative to the meandering field line, is therefore not diffusive relative to the mean magnetic field direction until the cross-field distance that is due to integration of Eq. (A.3) is comparable to that due to the integration of Eq. (8). At early times, the perpendicular pseudo-particle propagation is therefore only weakly stochastic with respect to the mean magnetic field, making our model consistent with the results presented by Laitinen et al. (2013). 